\title{
Celiac disease in the Mediterranean area
}

\author{
Francesca Tucci', Luca Astarita' ${ }^{1}$, Abdelhak Abkari², Mona Abu-Zekry ${ }^{3}$, Thomas Attard ${ }^{4}$, Mongi Ben Hariz ${ }^{5}$, \\ José Ramon Bilbao ${ }^{6,21}$, Ghazalia Boudraa ${ }^{7}$, Samir Boukthir ${ }^{8}$, Stefano Costa ${ }^{9}$, Veselinka Djurisic ${ }^{10}$, Jean-Pierre Hugot ${ }^{11}$, \\ Iñaki Irastorza ${ }^{12,21}$, Aydan Kansu ${ }^{13}$, Sanja Kolaček ${ }^{14}$, Giuseppe Magazzù ${ }^{9}$, Dušanka Mičetić-Turk ${ }^{15}$, Zrinjka Misak ${ }^{14}$, \\ Eleftheria Roma ${ }^{16}$, Pasqualino Rossi $^{17}$, Selma Terzic $^{18}$, Virtut Velmishi ${ }^{19}$, Carmela Arcidiaco ${ }^{1}$, Renata Auricchio ${ }^{1 \dagger}$ \\ and Luigi Greco ${ }^{1,20^{*}+}$
}

\begin{abstract}
Background: The World Gastroenterology Organization recommends developing national guidelines for the diagnosis of Celiac Disease (CD): hence a profile of the diagnosis of CD in each country is required. We aim to describe a cross-sectional picture of the clinical features and diagnostic facilities in 16 countries of the Mediterranean basin. Since a new ESPGHAN diagnostic protocol was recently published, our secondary aim is to estimate how many cases in the same area could be identified without a small intestinal biopsy.

Methods: By a stratified cross-sectional retrospective study design, we examined clinical, histological and laboratory data from 749 consecutive unselected CD children diagnosed by national referral centers.

Results: The vast majority of cases were diagnosed before the age of 10 (median: 5 years), affected by diarrhea, weight loss and food refusal, as expected. Only 59 cases (7.8\%) did not suffer of major complaints. Tissue transglutaminase (tTG) assay was available, but one-third of centers reported financial constraints in the regular purchase of the assay kits. 252 cases (33.6\%) showed tTG values over 10 times the local normal limit. Endomysial antibodies and HLA typing were routinely available in only half of the centers. CD was mainly diagnosed from small intestinal biopsy, available in all centers. Based on these data, only 154/749 cases (20.5\%) would have qualified for a diagnosis of CD without a small intestinal biopsy, according to the new ESPGHAN protocol.
\end{abstract}

Conclusions: This cross-sectional study of CD in the Mediterranean referral centers offers a puzzling picture of the capacities to deal with the emerging epidemic of CD in the area, giving a substantive support to the World Gastroenterology Organization guidelines.

Keywords: Mediterranean area, Celiac disease, World gastroenterology organization, ESPGHAN guidelines

\section{Background}

Celiac disease $(\mathrm{CD})$ increased at an unexpected rate in the last two decades [1-3]. It was long considered a problem limited to the wheat-consuming affluent societies of the western world [4], but it was recently reported in almost every wheat-consuming country worldwide [5]. In the near future, most $C D$ cases are expected to come from Africa, Asia and South America [6-9]. We estimate that

\footnotetext{
*Correspondence: ydongre@unina.it

${ }^{\dagger}$ Equal contributors

${ }^{1}$ European Laboratory for Food Induced Diseases, University of Naples Federico II, Naples 80131, Italy

${ }^{20}$ European Laboratory for Food-Induced Diseases, Department of Translational Medical Science, Section of Pediatrics, University of Naples Federico II, Via Sergio Pansini 5, 80131 Naples, Italy

Full list of author information is available at the end of the article
}

more than 5 million cases will occur in the Mediterranean region in the next 10 years. Without a timely diagnosis and appropriate health care, an excess mortality of more than 230,000 cases may be expected in the next decade [10]

Facilities and resources to control this modern noncommunicable disease epidemic are limited in many of the less affluent countries of the world including some of the Mediterranean countries. The World Gastroenterology Organization (WGO) recognized that the 'epidemic' of $\mathrm{CD}$ cannot be met without considering the local resources: hence it recommended adapting the diagnostic protocol according to available resources [11]. Recently, European Society for Paediatric Gastroenterology, Hepatology and Nutrition (ESPGHAN) published new diagnostic criteria for $\mathrm{CD}$ to simplify $\mathrm{CD}$ diagnosis by avoiding small bowel

\section{Biomed Central}


biopsy in selected patients [12,13], namely patients with clear symptoms, tissue transglutaminase (tTG) levels 10-fold above the upper limit of normal, positive antiendomysial antibodies (EMA) and specific HLA haplotype. This new algorithm, not requiring in all cases expensive endoscopy and pathology equipment, could facilitate the control over the coming CD epidemic in less privileged countries. The Mediterranean Network for the Management of Food-Induced Diseases (MEDICEL), which is an EUROMED-based action, to which all Mediterranean countries participate (Figure 1), aims at improving awareness of the forthcoming $\mathrm{CD}$ epidemic.

We aim to describe a cross-sectional picture of the clinical features and diagnostic facilities in 16 countries of the Mediterranean basin. Since the new ESPGHAN diagnostic protocol was recently published, our secondary aim is to estimate how many cases could be identified without a small intestinal biopsy in the same area.

\section{Methods}

Unfortunately Syria, Lebanon and Libya did not participate to the network. Palestine was not able to comply with the requirement for the study. Israel could not obtain the ethical permission to use data already collected for a similar study.

Each referral center from Albania, Algeria, Bosnia Herzegovina, Croatia, Egypt, France, Greece, Italy - Messina and Naples - Malta, Montenegro, Morocco, Slovenia, Spain, Tunisia and Turkey provided a list of 50 unselected consecutive $\mathrm{CD}$ cases diagnosed in the center by the best locally available methodologies. Using a standardized form, the clinical and laboratory data leading to the diagnosis were collected for each case. The methods used in each center for the serum assay of tTG were recorded, together with their local normal values. We reviewed the pathology procedure used to evaluate small intestinal biopsy specimens and recorded the methods used to evaluate the HLA haplotype. EMA assay was available in 8/16 centers: due to the subjectivity of the method, we could not obtain a standardized comparable set of results.

According to the WGO diagnostic protocol, tTG are an acceptable proxy of the EMA antibodies, especially in countries with limited resources ("The EMA test requires expert observers, and ELISA tests for detecting tTG antibodies should therefore be recommended in settings with low expertise") [11]. For these reasons, in the analysis, we did not considered the few available EMA values.

Based on the facilities and tests available in each center, for each case we estimated the probability of being diagnosed with $C D$ by the new ESPGHAN diagnostic criteria alone, without small intestinal biopsy. For each case we summed up the number of diagnostic criteria met (clinical symptoms, tTG above 10 folds the upper limit of normal and specific HLA). The sum of the criteria determined the final diagnostic score, which thus ranged from 1 to 3 . To reduce large tables, countries were often aggregated within their geographical region (Europe, Balkans, North Africa).

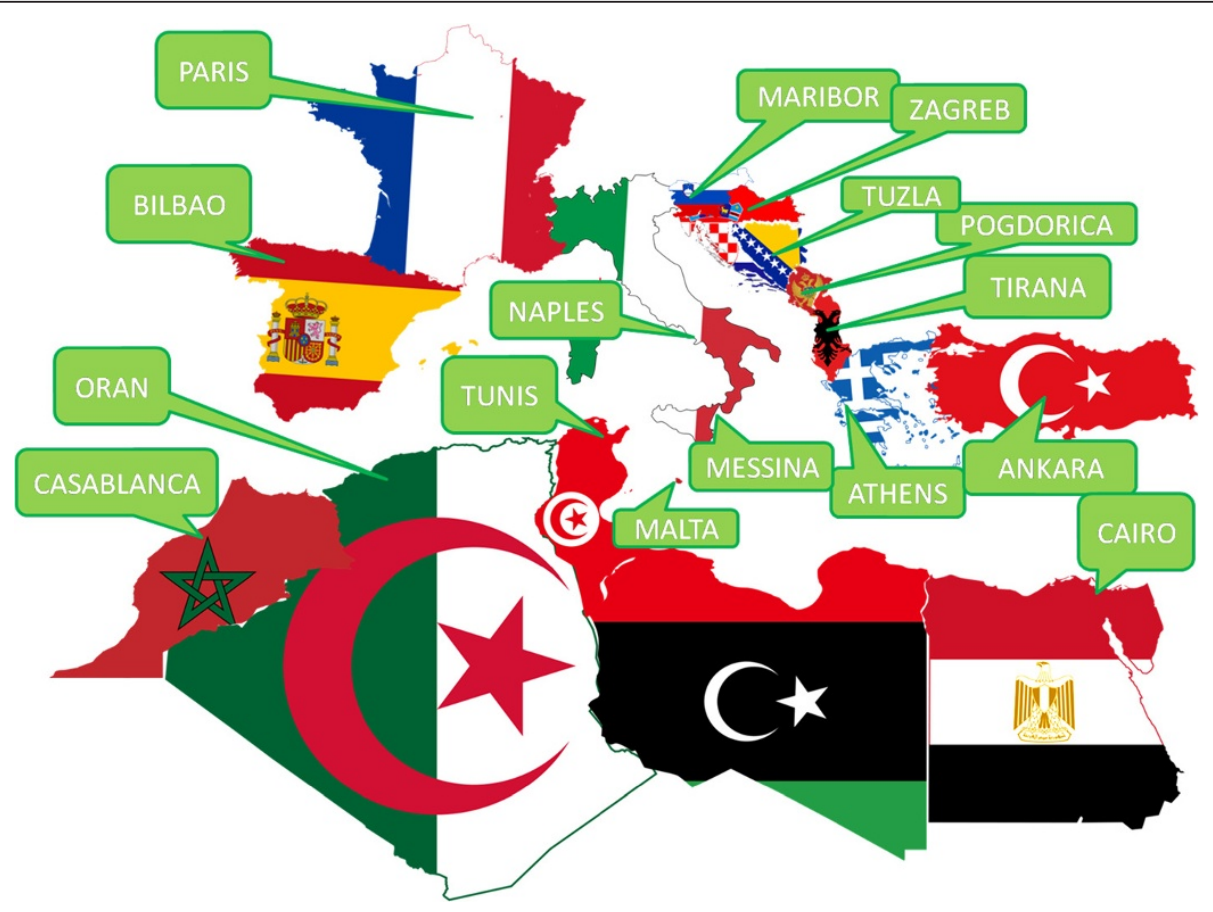

Figure 1 Map of referral centers in the participating countries. 
Data analysis was performed using the SPSS 16 software package (SPSS Inc., Chicago, IL, USA). Variables were $\log$ transformed when required because of their not normal distribution. Student's $t$ test and ANOVA were used to compare group means; non parametric tests were used when appropriate; $\mathrm{p}$ values $<0.05$ were considered significant.

\section{Ethics statement}

Institutional review boards at each collection site approved the study in each country (Albania, Algeria, Bosnia Herzegovina, Croatia, Egypt, France, Greece, Italy, Malta, Montenegro, Morocco, Slovenia, Spain, Tunisia and Turkey) (Additional file 1).

\section{Results}

A total of 800 forms were expected by the $16 \mathrm{CD}$ referral centers of the Mediterranean Basin, 50 each, but 51 (6.3\%) forms were excluded by the local centers because of missing data, thus 749 (93.7\%) CD cases were included in the analysis.

\section{Clinical presentation}

A total of $169 / 749$ patients (22.5\%) reported a case of CD among their first-degree relatives: mother in 44/749 (5.8\%); father in 45/749 (6\%); sibling in 45/749 (6\%); and more than one relative in $35 / 749(4.6 \%)$ cases. The female-to-male ratio was 1.6:1 (461 females; 288 males). Age at diagnosis ranged from 6 months to 18 years: 388 patients $(51.8 \%)$ were diagnosed within the first 5 years of life, and $23.4 \%$ during the first two years of life. The mean age at diagnosis was 5.97 years (SD 4.35; median 5 years). The distribution of age at diagnosis did not differ significantly (ANOVA $\mathrm{p}>0,1$ ) among the 3 geographical areas (Europe, Balkans and North Africa). Since the participants are referral centers, most patients had serious clinical complaints (Table 1). Clinical manifestations varied according to age (Table 2). Younger children (1-5 years old) more often suffered from

Table 1 Frequencies of symptoms in the three macro-areas

\begin{tabular}{llll}
\hline Symptoms & \multicolumn{3}{c}{ Area } \\
\cline { 2 - 4 } & Europe & Balkans & Africa \\
\hline Chronic diarrhea & $82(35.3 \%)$ & $149(44.5 \%)$ & $99(54.4 \%)$ \\
Weight loss & $81(34.9 \%)$ & $155(46.2 \%)$ & $67(36.8 \%)$ \\
Food refusal & $59(25.4 \%)$ & $107(31.9 \%)$ & $55(30.2 \%)$ \\
Vomiting & $122(52.6 \%)$ & $240(71.6 \%)$ & $113(62.1 \%)$ \\
Anemia & $22(9.5 \%)$ & $74(22.1 \%)$ & $31(17.0 \%)$ \\
Constipation & $11(4.7 \%)$ & $32(9.5 \%)$ & $8(4.4 \%)$ \\
Other & $13(5.6 \%)$ & $38(11.3 \%)$ & $18(9.9 \%)$ \\
No symptoms & $41(17.7 \%)$ & $3(0.9 \%)$ & $15(8.3 \%)$ \\
Total & 232 & 335 & 182 \\
\hline
\end{tabular}

Table 2 Presenting symptoms by ages

\begin{tabular}{llll}
\hline Symptoms & \multicolumn{3}{c}{ Age (years) } \\
\cline { 2 - 4 } & $\mathbf{0 - 5}$ & $\mathbf{6 - 1 0}$ & $\mathbf{1 1 - 1 8}$ \\
\hline Chronic diarrhea & $188(22.8 \%)$ & $87(17.9 \%)$ & $55(16.8 \%)$ \\
Weight loss & $156(18.9 \%)$ & $78(16 \%)$ & $69(21.1 \%)$ \\
Food refusal & $128(15.5 \%)$ & $63(13 \%)$ & $30(9.2 \%)$ \\
Vomiting & $221(26.8 \%)$ & $159(32.7 \%)$ & $95(29.1 \%)$ \\
Anemia & $67(8.1 \%)$ & $37(7.6 \%)$ & $23(7 \%)$ \\
Constipation & $26(3.2 \%)$ & $18(3.7 \%)$ & $7(2.1 \%)$ \\
Other symptoms & $22(2.7 \%)$ & $22(4.5 \%)$ & $25(7.6 \%)$ \\
No symptoms & $15(1.9 \%)$ & $22(4.5 \%)$ & $23(7 \%)$ \\
Total & $824(50.3 \%)$ & $486(29.7 \%)$ & $327(20 \%)$ \\
\hline
\end{tabular}

diarrhea and food refusal than older children. Vomiting, food refusal and growth impairment were common across all ages. Older children and adolescents ( $>10$ years) occasionally presented with extra-intestinal manifestations such as mood changes and anemia, but generally showed the same symptoms as younger children.

\section{Serology tests}

tTG data were available for 748 cases. The cutoff values of normality in the various countries ranged between 7 and $20 \mathrm{UI} / \mathrm{ml}$, and we normalized the reported raw values for the corresponding threshold of the center. The distribution of tTG values in times the normal local values shows that $252(36.6 \%)$ patients had values 10 times the normal or more and a considerable number, 103 (13.7\%), of CD cases were diagnosed by a tTG level not greater than twice the upper limit of the local normal values.

Table 3 Presence of the ESPGHAN diagnostic criteria in the $\mathbf{7 4 9}$ patients

\begin{tabular}{llll}
\hline Symptoms & & Frequency & Percentage \\
\hline \multirow{4}{*}{ tTG } & None & 59 & 7.9 \\
& Present & 685 & 91.4 \\
& & & \\
& $<10$ times & 496 & 66.2 \\
HLA & $>10$ times & 252 & 33.6 \\
& & & \\
& Absent & 432 & 57.7 \\
& Present & 317 & 42.3 \\
ESPGHAN diagnostic criteria & & \\
& 1 & 289 & 38.5 \\
& 2 & 294 & 39.2 \\
& 3 & 154 & 20.5 \\
\hline
\end{tabular}




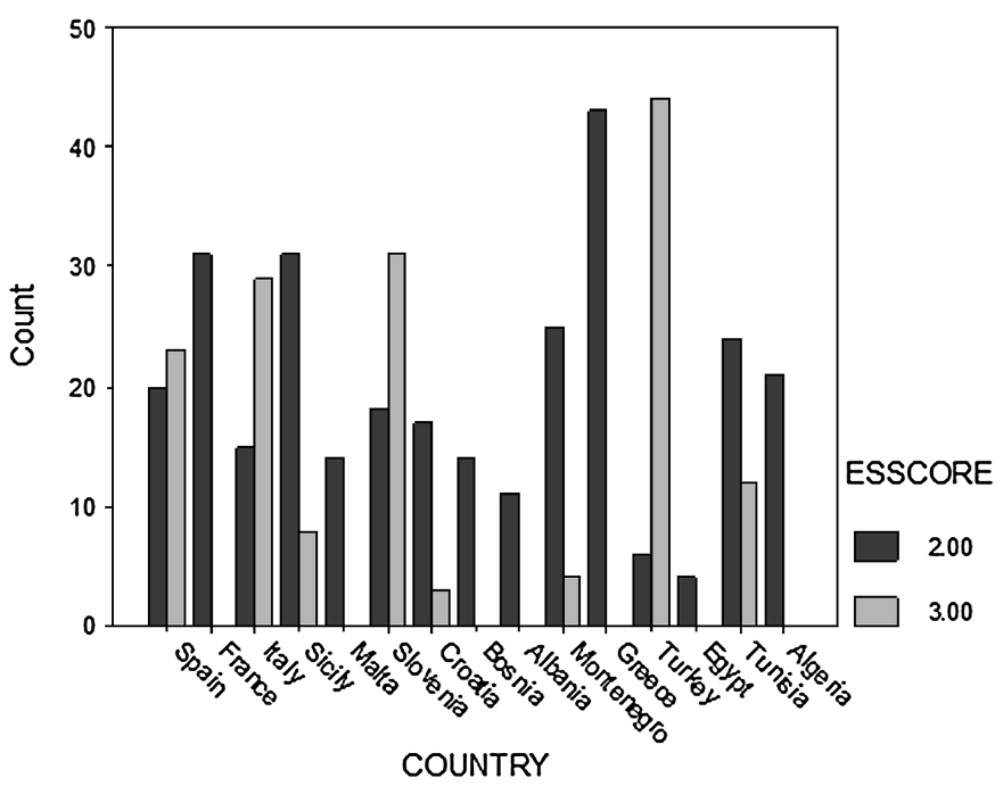

Figure 2 ESPGHAN score (ESSCORE) by country.

\section{Biopsy}

706/749 (94.2\%) CD patients underwent a duodenal biopsy. A biopsy report was not provided for 27/43 cases (62.8\%) in Albania, for 8/42 cases (19\%) in Bosnia Herzegovina, and for $4 / 36$ cases (11.1\%) in Egypt. Mild mucosal lesions (T0-T2) were found in 158 patients (22.4\%), while 56 (7.9\%) patients had partial atrophy (T3a), 492 (69.6\%) patients had more severe atrophy (T3b and T3c).

\section{HLA typing}

HLA typing was reported for all cases from Greece, Naples, Slovenia and Spain, in 14/42 of cases from Bosnia Herzegovina (33.3\%), in $13 / 50$ from Croatia (26\%), 16/50 from Messina (Sicily) (22\%) and 34/50 from Turkey (68\%). HLA results were missing in countries of North Africa (Algeria, Morocco, Tunisia and Egypt), Albania and Montenegro. In conclusion, 368/749 CD cases were genotyped for HLA (49.1\%). Of these 285 (77.4\%) carry the DQ2 heterodimer, 76 (20\%) the DQ8 heterodimer, whereas 7 (1.9\%) do not carry either of these molecules.

\section{When could the biopsy be omitted?}

Table 3 shows that $91.4 \%$ of cases had clear symptoms (criterion 1), 33.6\% had x10 tTG titers (criterion 2) and $42.3 \%$ of patients underwent HLA typing (criterion 3). When we examined the sum of criteria for each individual, we found that $39 \%$ of patients had clinical symptoms only, $40 \%$ had two criteria, i.e., clinical symptoms plus serum tTGx10 normal or HLA, and only 154 (20.7\%) of 749 patients had all 3 criteria thereby qualifying for a diagnosis without a biopsy. HLA typing was not available for half the cases; if we assume that all these cases had a compatible HLA, we may conclude that 304 (40.9\%) cases would qualify for the biopsy-free diagnosis.

Figure 2 shows the percentage of cases with 2 and 3 criteria in the various countries. It is clear that for many countries (Albania, Algeria, Bosnia Herzegovina, Egypt, France, Greece, Malta and Morocco) not one case would qualify for the biopsy-free protocol. In fact, it would be

\section{Table 4 Correlation between tTG and Marsh stages}

\begin{tabular}{lll}
\hline Country & Spearman Rho & p value \\
\hline Albania & 0.42 & 0.01 \\
Algeria & -0.14 & 0.32 \\
Bosnia & 0.09 & 0.58 \\
Croatia & -0.07 & 0.6 \\
Egypt & 0.17 & 0.3 \\
France & 0.06 & 0.6 \\
Greece & 0.007 & 0.9 \\
Malta & 0.18 & 0.9 \\
Montenegro & 0.25 & 0.079 \\
Morocco & 0.33 & 0.018 \\
Naples & 0.53 & 0.0001 \\
Sicily & 0.37 & 0.008 \\
Slovenia & 0.13 & 0.33 \\
Spain & -0.09 & 0.5 \\
Tunisia & 0.19 & 0.17 \\
Turkey & 0.21 & 0.16 \\
Total & 0.12 & 0.002 \\
\hline
\end{tabular}




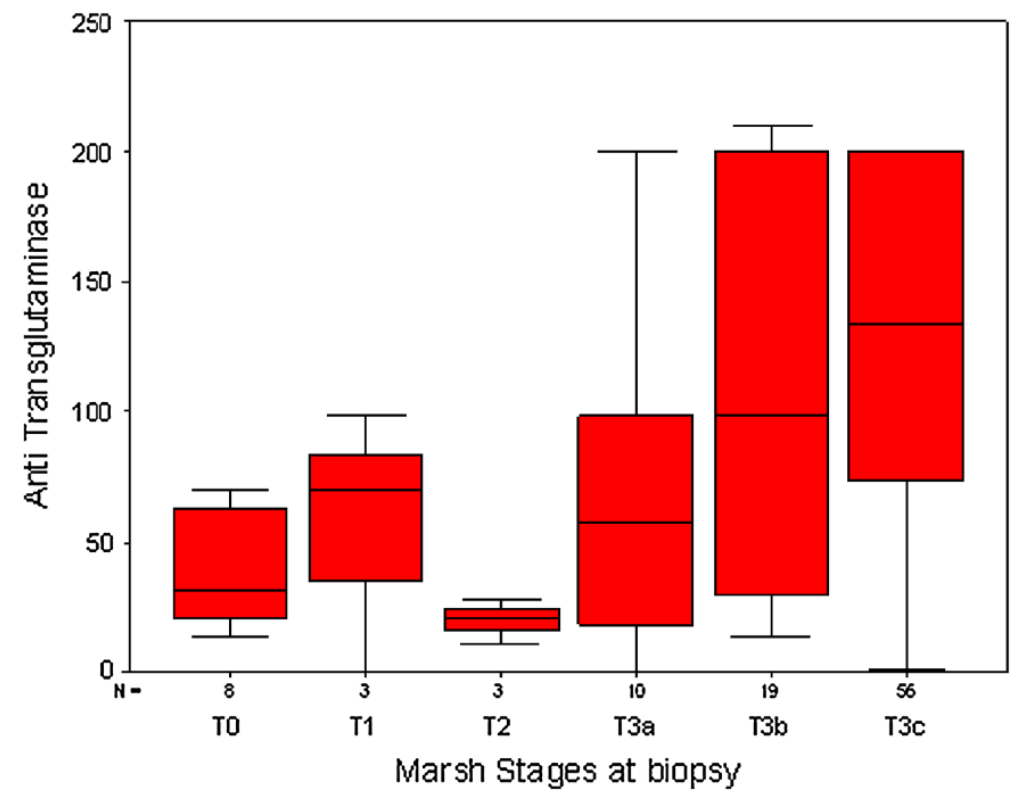

Figure 3 Median tTG levels by Marsh grading at biopsy (Messina and Naples, Italy).

applicable only for a sizeable number of cases from Italy, Slovenia, Spain, Tunisia and Turkey.

\section{Discussion}

Given the worldwide increase in $C D$ cases, there is an impelling need to simplify the diagnostic work-up to enable a timely diagnosis [11,12]. This is particularly true for countries that do not have access to sophisticated techniques. The WGO recommends evaluating the local availability of resources to develop a diagnostic protocol. We now provide a comprehensive picture of the diagnosis of CD in 16 Mediterranean countries. Being in European neighborhood area we evaluated whether the new ESPGHAN diagnostic protocol, which could potentially omit endoscopy and biopsy in a significant number of cases [13,14], could be applied efficiently in the 16 countries of the Mediterranean Basin who participate to the MEDICEL network. Indeed, in selected populations the "triple test" criteria appear to be helpful: a Spanish study [14] supports the view that in selected children who are symptomatic and positive for the triple test, $\mathrm{CD}$ diagnosis could be established independently of histological findings. Almost all cases here described were diagnosed by a small intestinal biopsy, while the aim of the new ESPGHAN criteria is to reduce the requirement for such and invasive procedures. We shared among the 16 partners the importance of avoiding, at least in a percentage of cases, such an invasive technique.

Unfortunately, in our setting only $20 \%$ of suspected $\mathrm{CD}$ cases with clear clinical symptoms might qualify for a CD diagnosis without a small intestinal biopsy. This percentage would have increased to about $40 \%$ if HLA testing had been available. This limitation is not confined to less equipped countries, but it also applies to many European centers with a long experience in $\mathrm{CD}$ diagnosis.

The chance of avoiding a small intestinal biopsy is based on the strength of the correlation between the tTG and the stage of mucosal damage. Recently Wakim-Fleming J et al. [15] suggested that titres of IgA tTG $>118$ IU identified patients with $\mathrm{CD}$ with a $2 \%$ false-positive rate. Titres of 21-118 UI, in combination with EMA dilution titer $\geq 1: 160$, had a positive predictive value of $83 \%$ for CD. Popp et al. [16] also found that in diabetic patients high tTG titres predict a diagnosis of $\mathrm{CD}$, when associated to EMA positivity. The relationship between antibodies and biopsy is crucial: we show on Table 4 the Spearman Correlation Coefficients between the values of the tTG(log) and the Marsh stages at biopsy in each country. It is clear that the correlation is strong and significant only in Italy (Messina and Naples), while it is not significant in all other countries. The basic assumption that tTG antibodies predict severe mucosal damage is not confirmed in this set of data. The shape of the correlation in Italy (Messina and Naples) shows that tTG does increase with Marsh Stages (Spearman Rho $=0.44$ ), but there are very ample 95\% Confidence Intervals around the median at all Marsh stages, making the basic assumption quite fragile also in this country (Figure 3). Based on our data, tTG does not appear to be actually a proxy of the degree of mucosal damage as evaluated by the Marsh score. Also in Italy, where there is a clear correlation, the interquartile range is very large, making this correlation unsafe to predict the mucosal damage by the high levels of antibody production. Although the correlation between the 
mucosal damage, estimated by the Marsh stages, and the level of tTG antibodies has been confirmed in several relevant studies [13], this correlation has several weakness, due to the nature of the data. tTG antibodies, as all antibodies, do not have a normal distribution, but a logarithm distribution and Marsh stages have an ordinal distribution, not a continuous one. So the relationship between these two variables is intrinsically prone to large or very large confidence intervals, which reflect not only the nature of the variables, but also the wide polymorphism of the phenotype of the disease. The quality of serological test and the scarcity of HLA testing are the critical points that, at present, limit the chance to diagnose $\mathrm{CD}$ without a biopsy.

Unfortunately, in several of the participating referral centers, the laboratory experience to assay the tTG is limited and performed only when financial resources are available. Some laboratories produce semi-quantitative data, increasing the uncertainty of the assessment. One of the objectives of the MEDICEL network is to support the upgrading of local diagnostic resources: hence we are now running ad hoc procedure to standardize the method of antibody assay and to increase the availability of HLA haplotyping by exploiting the new technologies that attempt to bring the test to the point of care $[17,18]$.

This cross-sectional study provides the first picture of the profile of $\mathrm{CD}$ in the countries studied and of the diagnostic resources available in the referral centers. Most cases were symptomatic showing the classical clinical profile. This will probably change significantly in the near future when awareness about $\mathrm{CD}$ increases, as has occurred in several European countries $[3,10]$. This study suffers from the objective limitation of being a retrospective study. Nevertheless, we needed a cross-sectional picture of the pattern of celiac disease in the area, for which this kind of study is rapidly informative. In the same area we have already started a prospective study in order to validate the findings of this actual study.

\section{Conclusion}

In conclusion, this cross-sectional survey provides a multifaceted picture of the $\mathrm{CD}$ domain in the Mediterranean area. Being aware of the expanding epidemic of $\mathrm{CD}$ over the wheat consuming populations, we hope that simplified diagnostic criteria, possibly avoiding the expensive biopsy, could help to diagnose cases outside the very few referral centers in developing countries. Unfortunately this study does not support this chance to date, but does identify the critical points to be met in order to expand the advantages of the new ESPGHAN diagnostic protocol, especially in countries that need this change the most. These results provide to each participant country required data to develop local strategies according to the WGO recommendations.

\section{Additional file}

Additional file 1: National Ethical Permissions.

Competing interests

The authors declare that they have no competing interests.

\section{Authors' contributions}

$F T, L A, R A$ and LG planned the study, developed the forms, run the analysis and lead the writing of the manuscript. PR, MBH, AK, SK, GM, ZM made substantial contributions to conception and design and acquisition of data. AA, MAZ, JRB, GB, SB, SC, VD, JPH, II, DMT, ER, ST, W, CA have been involved in drafting the manuscript and collecting data. All authors read and approved the final manuscript.

\section{Acknowledgements}

We thank the Italian Celiac Society (AIC) and the Association of European Coeliac Societies (AOECS) for their role in the coordination of the participating Mediterranean countries. We are grateful to Jean Ann Gilder (Scientific Communication srl) for writing assistance.

\section{Disclosure of funding}

This work was by sponsored by the Ministery of Health of Italy, Direction of International Affairs; Euromed (Episouth) action. Project: "Food-induced diseases - Celiac Disease (Medicel) - Phase II" (CUP n E61J11000450001). The funders had no role in study design, data collection and analysis, decision to publish, or preparation of the manuscript.

\section{Author details}

${ }^{1}$ European Laboratory for Food Induced Diseases, University of Naples Federico II, Naples 80131, Italy. ${ }^{2}$ Hôpital des Enfant IbnRochd de Casablanca, Casablanca 20050, Morocco. ${ }^{3}$ Children's Hospital, Gastrointestinal Unit, Cairo University, Cairo 12511, Egypt. ${ }^{4}$ Mater Dei Hospital, Msida MSD 2090, Malta. ${ }^{5}$ Paediatric Unit, Mongi SLIM's Hospital of Tunis, Marsa 2078, Tunisia.

${ }^{6}$ Endocrinology, Diabetes and Nutrition Research Group, Hospital de Cruces, Barakaldo-Bizkaia, Basque Country 48903, Spain. ${ }^{7}$ Clinique Amilcar Cabral, Oran 31026, Algeria. ${ }^{8}$ Hôpital d'enfants, Tunis, Tunisia. ${ }^{9}$ Regional Celiac Center, University Hospital G. Martino, Messina 98125, Italy. ${ }^{10}$ Clinical Centre of Montenegro, Institute for Children s Disease, Podgorica, Montenegro.

${ }^{11}$ UMR843, INSERM, Assistance Publique Hopitaux de Paris et Université, Paris Diderot, Paris, France. ${ }^{12}$ Pediatric Gastroenterology, Hospital de Cruces, Barakaldo 48903, Spain. ${ }^{13}$ Faculty of Medicine, Department of Pediatric Gastroenterology, Ankara University, Ankara 06100, Turkey. ${ }^{14}$ Children's Hospital Zagreb, Zagreb 10000, Croatia. ${ }^{15}$ University Medical Centre Paediatric Department, Ljubljanska, Maribor 2000, Slovenia. ${ }^{16}$ Aghia Sophia Children's Hospital, Athens University, Goudi, Athens 11527, Greece. ${ }^{17}$ International Affairs Direction, Ministry of Health, Rome, Italy. ${ }^{18}$ Department of Children Diseases, University Clinical Center, Tuzla, Bosnia Herzegovina. ${ }^{19}$ Gastrohepatology of University Hospital Centre "Mother Teresa", 1000 Tirana, Albania. ${ }^{20}$ European Laboratory for Food-Induced Diseases, Department of Translational Medical Science, Section of Pediatrics, University of Naples Federico II, Via Sergio Pansini 5, 80131 Naples, Italy. ${ }^{21}$ BioCruces Research Institute, Cruces University Hospital, University of the Basque Country (UPV-EHU), Barakaldo, Basque Country, Spain.

Received: 18 July 2013 Accepted: 30 January 2014 Published: 11 February 2014

\section{References}

1. Green PH, Cellier C: Celiac disease. N Engl J Med 2007, 357:1731-1743.

2. Di Sabatino A, Corazza GR: Coeliac disease. Lancet 2009, 373:1480-1493.

3. Lohi S, Mustalahti K, Kaukinen K, Laurila K, Collin P, Rissanen H, Lohi O, Bravi E, Gasparin M, Reunanen A, Mäki M: Increasing prevalence of coeliac disease over time. Aliment Pharmacol Ther 2007, 26:1217-1225.

4. Dubé C, Rostom A, Sy R, Cranney A, Saloojee N, Garritty C, Sampson M, Zhang L, Yazdi F, Mamaladze V, Pan I, Macneil J, Mack D, Patel D, Moher D: The prevalence of celiac disease in average-risk and at-risk Western European populations: a systematic review. Gastroenterology 2005, 128(4 Suppl 1):S57-S67. C. 
5. Byass $P$, Kahn $K$, Ivarsson $A$ : The global burden of childhood coeliac disease: a neglected component of diarrhoeal mortality? PLoS One 2011, 6:e22774.

6. Cummins AG, Roberts-Thomson IC: Prevalence of celiac disease in the Asia-Pacific region. J Gastroenterol Hepatol 2009, 24:1347-1351.

7. Wu J, Xia B, von Blomberg BM, Zhao C, Yang XW, Crusius JB, Peña AS: Coeliac disease: emerging in China? Gut 2010, 59:418-419.

8. Barada K, Bitar A, Mokadem MA, Hashash JG, Green P: Celiac disease in Middle Eastern and North African countries: a new burden? World J Gastroenterol 2010, 16:1449-1457.

9. Malekzadeh R, Sachdev A, Fahid AA: Coeliac disease in developing countries: Middle East, India and North Africa. Best Pract Res Clin Gastroenterol 2005, 19:3518

10. Greco L, Timpone L, Abkari A, Abu-Zekry M, Attard T, Bouguerrà F, Cullufi P, Kansu A, Micetic-Turk D, Mišak Z, Roma E, Shamir R, Terzic S: Burden of celiac disease in the Mediterranean area. World J Gastroenterol 2011, 17:4971-4978.

11. Bai JC, Fried M, Corazza GR, Schuppan D, Farthing M, Catassi C, Greco L, Cohen H, Ciacci C, Eliakim R, Fasano A, González A, Krabshuis JH, LeMair A: World gastroenterology organization global guidelines on celiac disease. J Clin Gastroenterol 2013, 47:121-126.

12. Ribes-Koninckx C, Mearin ML, Korponay-Szabó IR, Shamir R, Husby S, Ventura A, Branski D, Catassi C, Koletzko S, Mäki M, Troncone R, Zimmer KP, ESPGHAN Working Group on Coeliac Disease Diagnosis: Coeliac disease diagnosis: ESPGHAN 1990 criteria or need for a change? Results of a questionnaire. The ESPGHAN Working Group on Coeliac Disease Diagnosis. J Pediatr Gastroenterol Nutr 2012, 54:15-19.

13. Husby S, Koletzko S, Korponay-Szabó IR, Mearin ML, Phillips A, Shamir R, Troncone R, Giersiepen K, Branski D, Catassi C, Lelgeman M, Mäki M, Ribes-Koninckx C, Ventura A, Zimmer KP, ESPGHAN Working Group on Coeliac Disease Diagnosis, ESPGHAN Gastroenterology Committee, European Society for Pediatric Gastroenterology, Hepatology, and Nutrition: European Society for Pediatric Gastroenterology, Hepatology, and Nutrition guidelines for the diagnosis of coeliac disease. J Pediatr Gastroenterol Nutr 2012, 54:136-160.

14. Klapp G, Masip E, Bolonio M, Donat E, Polo B, Ramos D, Ribes-Koninckx C: Coeliac disease: the new proposed ESPGHAN diagnostic criteria do work well in a selected population. J Pediatr Gastroenterol Nutr 2013, 56:251-256.

15. Wakim-Fleming J, Pagadala MR, Lemyre MS, Lopez R, Kumaravel A, Carey WD, Zein NN: Diagnosis of celiac disease in adults based on serology test results, without small-bowel biopsy. Clin Gastroenterol Hepatol 2013, 11:511-516.

16. Popp A, Mihu M, Munteanu M, Ene A, Dutescu M, Colcer F, Raducanu D, Laurila K, Anca I, Mäki M: Prospective antibody case finding of coeliac disease in type-1 diabetes children: need of biopsy revisited. Acta Paediatr 2013, 102:e102-e106.

17. Bienvenu F, BessonDuvanel C, Seignovert C, Rouzaire P, Lachaux A, Bienvenu J: Evaluation of a point-of-care test based on deamidatedgliadin peptides for celiac disease screening in a large pediatric population. Eur J Gastroenterol Hepatol 2012, 24:1418-1423.

18. Giersiepen K, Lelgemann M, Stuhldreher N, Ronfani L, Husby S, Koletzko S, Korponay-Szabó IR, ESPGHAN Working Group on Coeliac Disease Diagnosis: Accuracy of diagnostic antibody tests for coeliac disease in children: summary of an evidence report. J Pediatr Gastroenterol Nutr 2012, 54:229-241.

\section{Submit your next manuscript to BioMed Central and take full advantage of:}

- Convenient online submission

- Thorough peer review

- No space constraints or color figure charges

- Immediate publication on acceptance

- Inclusion in PubMed, CAS, Scopus and Google Scholar

- Research which is freely available for redistribution

Submit your manuscript at www.biomedcentral.com/submit
Ciomed Central 